\title{
The Role of CGRP Inhibition and High-Intensity Interval Training (HIIT) on Mtorc2 and VEGF Gene Expression in The Hippocampal Tissues of Male Wistar Rats
}

\section{Asma Jafari Khoshan-Abadi}

University of Sistan and Baluchestan

\section{Shila Nayebifar}

University of Sistan and Baluchestan

Soheil Aminizadeh ( $\nabla$ soheilaminizadeh@gmail.com )

Kerman University of Medical Sciences https://orcid.org/0000-0003-3651-3505

Majid Vahidian-Rezazadeh

University of Sistan and Baluchestan

\section{Research Article}

Keywords: High-Intensity Interval Training (HIIT), CGRP, VEGF, mTORC-2

Posted Date: February 25th, 2022

DOI: https://doi.org/10.21203/rs.3.rs-1240960/v1

License: (c) (1) This work is licensed under a Creative Commons Attribution 4.0 International License.

Read Full License 


\section{Abstract \\ Background}

The hippocampus, as one of the most critical regions of the brain, is significantly affected by exercise. The primary purpose of this study is to evaluate the effect of six weeks of high-intensity interval training (HIIT) and inhibition of calcitonin gene-related peptide (CGRP) on gene expression of vascular endothelial growth factor (VEGF) and serine/threonine type 2 kinase protein (mTORC2) in hippocampal tissue of male Wistar rats.

\section{Methods}

Thirty-two healthy male Wistar rats (8 weeks of age) were randomly assigned to one of four groups: control, CGRP inhibition, HIIT+CGRP inhibition, or HIIT. The CGRP inhibition group received 0.25 $\mathrm{mg} / \mathrm{kg} / \mathrm{day}$ of antibody intraperitoneally. The animals in the training groups underwent high-intensity interval training (HIIT, ten 2-minute work bouts per day at $22 \mathrm{~m} / \mathrm{min}$ interspersed by 2-minute rest periods, five days/week). Real-time PCR (2- $\Delta \Delta \mathrm{CT})$ and one-way ANOVA were employed to measure the expression of genes and analyze the data, respectively.

\section{Results}

HIIT significantly enhanced VEGF $(p \leq 0.002)$ and mTORC2 $(p \leq 0.006)$ gene expression in the hippocampal tissue compared to the control group. HIIT+CGRP inhibition resulted in a substantial increase in VEGF expression compared to the control group $(\mathrm{p} \leq 0.007)$. While HIIT+CGRP inhibition significantly lowered mTORC2 gene expression ( $p \leq 0.001)$, HIIT alone did not $(p \leq 0.001)$.

\section{Conclusions}

HIIT-induced physiological alterations in the hippocampus can improve the brain's functional status via upregulation of VEGF and mTORC2 gene expression as an effective non-pharmacological method. Additionally, HIIT+CGRP inhibition may represent a new approach via the VEGF and mTORC2 pathways in the hippocampus.

\section{Background}

Exercise training directs gene expression toward patterns necessary for survival (1). As an effective strategy, high-intensity interval training (HIIT) helps strengthen the central nervous system (2) and improves physical readiness (3). It results in a distinct gene expression pattern in different brain regions, with considerable alterations in associated genes (4). 
Inactivity has long been a significant risk factor for brain function, including the hippocampus, which is a brain structure that enhances memory and cognitive performance (5). Physical activity mitigates the deterioration in brain function and strengthens the brain's connections and hippocampus-cortical network. Hence, the extent to which the hippocampal network changes in this functional pattern is critical, as exercise exerts the most substantial impact on the hippocampus structure (6). Improvements in neurogenesis, protection, angiogenesis, and nerve flexibility are some of the exercise-induced neurological changes in the hippocampus (7), which result in viability via cell proliferation and apoptosis inhibition (8). As a result, these exercise-induced factors have a significant impact on brain structure and growth (9) and can regulate a wide variety of physiological changes in involved neurotropic factors (10).

Neuropeptides such as calcitonin gene-related peptide (CGRP) and its receptors appear to be dispersed in the hippocampus (CA3 pyramidal cells (Region III of the hippocampus)) and dentate gyrus granule cells (11), which are involved in normal brain development (12). CGRP is a regulatory neuropeptide (13) that increases anti-apoptotic signaling in the brain, particularly in the hippocampus. It protects the endothelium and promotes neurogenesis and angiogenesis $(14,15)$. The effect of CGRP on endothelium includes growth factor signaling (14), where CGRP inhibition can increase inflammation and decrease the expression of vascular endothelial growth factor (VEGF) (16). VEGF can be produced in a variety of cells and the dentate gyrus of the hippocampus (9). It communicates with endothelial cells via VEGF-R2 receptors (17). Induced by hypoxia (18), the protein plays a critical role in angiogenic and neurogenic processes. Indeed, VEGF increase is one of the protective mechanisms promoted by CGRP (15-18). VEGF, which is required for neuroprotective signaling (19), protects endothelial cells by triggering anti-apoptotic genes and protective signaling pathways via PI3K/AKT (Protein kinase B (PKB)) and thereby controls endothelial survival (14). The functions of numerous neutrophilic factors in the brain overlap during growth, and findings indicate that hippocampal neurons' vitality depends on growth factors such as VEGE and mTORC2. mTORC2 regulates cell proliferation induced by growth stimuli and serves as a connector for several signaling pathways (20). Additionally, it plays a critical role in neuronal morphology and synaptic function. It is abundantly produced in the brain, particularly in nerve cells, and is vital for multiple aspects of hippocampus growth and function (21).

Exercise intensity is a critical factor in determining gene expression (9). Lactate and hypoxia, a byproduct of intensive exercise, have been demonstrated to influence certain effects of exercise on the brain (22). Exercise effects may be linked with viability, which induces VEGF production by activating the PI3K/AKT signaling pathway (23). Recent data indicate that physical activity regulates VEGF levels by activating the mTOR complex pathway (24). As a result, it may affect the physical activity of the VEGF and mTORC2 genes via signaling mechanisms in the rats' hippocampus (24).

Numerous studies have been conducted on the impact of HIIT on skeletal muscles and heart tissues in disease and health conditions. However, the literature has not well investigated the effect of such exercise on brain tissue adaptations and the effective rate of these exercises on VEGF and mTORC2 levels, as factors involved in the process of angiogenesis and nerve support in various regions of the brain. Thus, the following concerns are raised: 1 . Is HIIT an effective method of maintaining brain health as a time- 
efficient alternative to regular aerobic exercise in terms of its influence on growth factors such as VEGF and mTORC2, and 2. Can the inhibition of CGRP have any impact on VEGF and mTORC2? Thus, this study aimed to investigate the extent to which changes in VEGF and mTORC2 gene expression in the hippocampal region following six weeks of HIIT+CGRP inhibition would affect the health of male Wistar rats' brain tissue.

\section{Methods}

\section{Animals}

Thirty-two male Wistar rats were purchased from the Physiology Research Center affiliated with Kerman University of Medical Sciences. The rats (8 weeks of age and weighing $205 \pm 15 \mathrm{~g}$ ) were housed in a $12: 12$ dark-light cycle at a temperature of $22 \pm 2^{\circ} \mathrm{C}$, relative humidity of $55 \%$, and free access to water and food (Pellet, Javaneh Khorasan Company). The rats were randomly assigned to one of four groups ( $n=8$ per group): control, CGRP inhibition, HIIT+CGRP inhibition, or HIIT training alone. The ethical principles of the study followed the principles of working with laboratory animals (IR.USB.REC.1400.029).

\section{Training Protocol}

Training Protocol

Rats retained familiarity at a speed of $15 \mathrm{~m} / \mathrm{min}$ for 15 minutes for two weeks. The exercise training intensity was determined by measuring blood lactate levels immediately after exercise using a lactometer (Lactate Scout Company/Code: 37, Germany), with values above $6 \mathrm{mmol} / \mathrm{L}$ deemed high intensity (25). The speed test was initiated with a $10 \mathrm{~m} / \mathrm{min}$ warm-up that gradually increased $(0.3 \mathrm{~m} / \mathrm{min})$ until exhaustion (26). Ten two-minute work bouts were held daily at approximately $22 \mathrm{~m} / \mathrm{min}, 29^{\circ}$ slope, interspersed by two-minute rest periods, five days per week for six weeks (27). The control and CGRP inhibition groups were not trained during the experiment and were kept fixed on the conveyor for each session to induce treadmill stress.

\section{Cgrp Inhibition}

A daily dose of $0.25 \mathrm{mg} / \mathrm{kg}$ CGRP antibody (BIBN4096BS - Merck Company, Germany) (28) was administered intraperitoneally to inhibit CGRP. The injection was performed in the HIIT+CGRP inhibition 3 hours before performing the protocol. As the CGRP antibody was used intraperitoneally, we were unable to control the inhibitor's global effects on other tissues. To ensure CGRP inhibition, we evaluated CGRP gene expression in the hippocampus tissue after the protocol concluded (Fig. 1.).

\section{Sampling}


Forty-eight hours after the last training session, all four groups were anesthetized intraperitoneally with ketamine $(10 \mathrm{mg} / \mathrm{kg})$ and xylazine $(90 \mathrm{mg} / \mathrm{kg})$, and hippocampus tissue was immediately removed and frozen in liquid nitrogen $\left(-80^{\circ} \mathrm{C}\right)$ for further measurements.

\section{Extraction Protocol}

cDNA synthesis and real-time PCR

The tissues were first dissected into tiny pieces and powdered in liquid nitrogen using a mortar and pestle. Afterward, $10-20 \mathrm{mg}$ of powdered tissue was added to $0.3 \mathrm{ml}$ of RLT buffer. The mixture was then thoroughly mixed with $0.59 \mathrm{ml}$ RNase-free water and incubated for 10 minutes at $55^{\circ} \mathrm{C}$ in a dry bath. Subsequently, the resulting mixture was centrifuged for 3 minutes at $12000 \mathrm{~g}$ in a refrigerated centrifuge apparatus at $4^{\circ} \mathrm{C}$. Thereafter, the supernatant was transferred to a tube devoid of RNase $(1.5 \mathrm{ml})$. Following that, $0.5 \mathrm{ml}$ of RPE solution was added to the column, which was centrifuged at room temperature for one minute at $12000 \mathrm{~g}$ to remove any remaining materials. The extracted RNA was then used to initiate the CDNA synthesis stage. CDNA was created based on the purity of the extracted RNA and the cDNA synthesis protocol. Real-time PCR including primary Denaturation in $95^{\circ} \mathrm{C}$ and for 10 minutes ${ }^{\circ} \mathrm{C}$ in each PCR cycle in $95^{\circ} \mathrm{C}$ for $15 \mathrm{~s}$ and regarding the primers annealing temperature, each cycle was considered 30 s (40 cycles). $18 \mathrm{~S}$ was used as internal control and the expression rate of the considered genes was measured with 2- $\Delta \Delta \mathrm{CT}$. Table 1 presents the primer sequences (29).

Table 1

Primers sequences employed for gene expression measurements using real-time PCR

\begin{tabular}{|llll|}
\hline Genes & Forward primer $\left(\mathbf{5}^{\prime} \rightarrow \mathbf{3}^{\prime}\right)$ & Reverse primer $\left(\mathbf{5}^{\prime} \rightarrow \mathbf{3}^{\prime}\right)$ & $\begin{array}{l}\text { PubMed } \\
\text { Accession }\end{array}$ \\
\hline 18S & GCAATTATTCCCCATGAACG & GGCCTCACTAAACCATCCAA & 7MQ9_L1 \\
\hline mTORC2 & GGTGGACGAGCTCTTTGTCA & AGGAGCCCTAACACTCGGAT & XM_032894667.1 \\
VEGF & GTCACCACCACACCACCATCGT & CTCCTCTCCCTTCATGTCAGGCT & XM_032900655.1 \\
\hline CGRP & CTGGAG CAGGAGCAAGAGAG & TGAGTCA CACAGGTGGCAGT & NM_001101.3 \\
\hline
\end{tabular}

\section{Statistical Analysis}

Data were expressed as mean and standard deviation (SPSS software, version 22). Shapiro-Wilk test was used to determine the normality of data distribution, and one-way analysis of variance and Tukey's post hoc test were utilized to analyze the data. The significance level was considered $P \leq 0.05$.

\section{Results}

The effect of HIIT on VEGF and mTORC2 gene expression 
VEGF and mTORC2 expression levels were significantly increased in the HIIT group compared to the control group ( $P=0.002$ and $P=0.006$, respectively). In contrast, the mTORC2 mRNA level in hippocampal tissue was significantly decreased in the CGRP inhibition group and HIIT+CGRP inhibition group compared to the control group ( $P=0.001)$ (Fig. 2).

VEGF and mTORC2 expressions were significantly increased in the HIIT group compared to the control group ( $P=0.002$ and $P=0.006$, respectively). On the contrary, the mTORC2 mRNA level in the hippocampal tissue was decreased significantly in the CGRP inhibition and HIIT+CGRP inhibition groups compared to the control group $(P=0.001)$ (Fig. 2).

The combined effect of HIIT and CGRP inhibition on VEGF and mTORC2 gene expression

VEGF level was significantly higher in the HIIT+CGRP inhibition group than in the control group. There was a significant difference in MTORC2 between the HIIT+CGRP inhibition groups and the HIIT group $(P=0.001)$, with the mTORC2 being lower in the HIIT+CGRP inhibition group than in the HIIT group (Fig. 3.).

\section{Discussion}

The current findings provide pioneering evidence in support of the fact that CGRP and high-intensity interval training increase VEGF and mTORC2 mRNA levels in the hippocampus of healthy male rats. Additionally, our research establishes that HIIT enhances cell proliferation, possibly via CGRP signaling. Specifically, our findings indicate that 1) exercise training can enhance VEGF and mTORC2 expression in brain tissue and 2) CGRP and mTORC2 may interact in the hippocampus.

Similar to the present study, Lu et al. measured the effect of eight weeks of intense exercise intervention higher than lactate threshold on VEGF levels in the brain tissue of C57BL/ 6 rats (22). They discovered an increase in VEGF mRNA levels. Lactate generated by muscles appears to enter the brain via MCT (monocarboxylate transporters), where it amplifies VEGF mRNA expression in brain tissue (22). In this context, it has been demonstrated that CGRP secretion is directly associated with lactate generation and linearly related to lactate release during hypoxic exercise. Given that CGRP is positively linked with VEGF release, acute exercise may cause an increase in VEGF content by raising CGRP $(30,31)$. Rezaei and Nourshahi's experiments also revealed the usefulness of reducing the rate of brain stroke related to higher VEGF levels in the brain tissue of male Wistar rats (32). When exposed to anoxia, high-intensity intermittent exercise may decrease cerebral blood flow, reduce oxygenation and delivery to the brain, and considerably increase VEGF expression. This contributes to brain recovery following a stroke (10). Increased blood-brain barrier permeability is another stimulus, as demonstrated in a study by Cotman et al., where VEGF increased in the periphery crossed the blood-brain barrier and entered the brain, resulting in an increasing effect of cerebral VEGF (33). The inverse response of exercise intensity to VEGF expression (10) may be explained by the impact of high-intensity exercise on glucose uptake in the brain, as high-intensity exercise reduces glucose uptake in the brain (9). Collectively, the current findings demonstrate that part of the beneficial interventional effects of intensive intermittent exercise might be 
attributed to increased angiogenesis and neurogenesis in the hippocampus of healthy male rats via positive regulation of VEGF.

Despite this study's increase in mTORC2 expression, certain mechanisms have been postulated to explain why mTORC2 function improves during intense intermittent exercise. Growth factor signals trigger this complex, and VEGF has been shown to activate several downstream pathways involved in angiogenesis, including the PI3K-Akt/mTORC2 pathway (34). When growth factor signals activate mTORC2, the increase in mTORC2 may be followed by an increase in VEGF due to intense activity. Additionally, exercise increases AKT phosphorylation in Ser473 in the dentate gyrus of the hippocampus (30). mTORC2 mediator has established the role of AKT ser473 phosphorylation (35). Therefore, mTORC2 may be directly related to AKT. In sum, mTORC2 integrates growth signals via a variety of mechanisms that regulate cell viability (36). The current work on CGRP inhibition and HIIT contradicts the studies by Mitsuaka et al. (2018) (37) and Burkham's (2019) (14), which revealed that CGRP was directly involved in the production of VEGF by the endothelium because HIIT+CGRP inhibition on VEGF levels was accompanied with VEGF increase. This inconsistency is most likely owing to the fact that VEGF is produced by alternate routes, and it is expected that exercise-induced changes will be more pronounced on these pathways. However, the decrease in MTORC2 expression appears to increase VEGF expression, as Tian et al. (38) have shown. They demonstrated that CGRP inhibition had a negative effect on mTOR signaling in brain tissues, while these changes in FoxO3a (Forkhead box 03) levels were quite the opposite. Hence, the FoxO3a rise had a downtrend after CGRP injection. As a transcription factor, FoxO3a is affected by mTORC2 (39), which may be relevant to the expression of FoxO3a-suppressed VEGF (40). This is indeed not parallel with the present study findings. Interestingly, CGRP plays an important role in eliminating apoptosis and autophagy by activating Akt/mTORC2 signaling, which indicates cell proliferation and viability in brain tissue (39). In contrast to the increase in VEGF, it appears that the current study's absence of mTORC2 increase can be detected in several stress-sensitive regions of the brain, including the hippocampus. As a result, forced treadmill running might be viewed as a source of stress generation (41).

There have been no studies to date that examine the concurrent effect of HIIT and CGRP inhibition on mTORC2 and VEGF levels in hippocampus tissue. However, this work demonstrated that inhibiting CGRP alters mTORC2 expression, implying a mediating role for CGRP in the process of mTORC2 production. Thus, in order to capture more precise cellular mechanisms, future research should include experimental studies on animal species and tissue types. The study's primary limitation was the time period between animal sacrifices, as VEGF and mTORC2 are time-dependent.

\section{Conclusion}

HIIT triggers an interactive set of growth factor signaling. Thus, six weeks of HIIT and inhibition of CGRP may substantially affect the expression of VEGF and mTORC2 genes in the hippocampus tissue of male Wistar rats. As such, CGRP can be assumed to be a significantly positive factor in hippocampal tissue. 


\section{Abbreviations}

Calcitonin gene-related peptide; CGRP

Serine/threonine type 2 kinase protein; mTORC2

High-intensity interval training; HIIT

Phosphoinositide 3-kinases; PI3K

Protein kinase B; PKB

\section{Declarations}

\section{Ethics approval and consent to participate}

The current study was approved by the ethics committee of Sistan and Baluchestan University (IR.USB.REC.1400.029).

\section{Consent for publication}

Not Applicable.

\section{Availability of data and materials}

Not Available.

\section{Competing interests}

The authors confirm that there is no conflict of interest associated with this publication.

\section{Funding information}

This study was supported and funded by Sistan and Baluchestan University. Sistan and Baluchestan University provided financial support for the conduct of the research and preparation of the article, in study design, in the collection, analysis and interpretation of data.

\section{Author's contribution statement}

AJKA and SA derived the hypothesis and conceived the study design, planned and organized the data collection; SA performed the laboratory analyses, collected the data, and planned and performed the statistical analyses; SA, SN, and MVR wrote the manuscript. This report was critically reviewed and subsequently approved by all authors.

\section{Acknowledgments}

We would like to express our gratitude to the laboratory personnel at Kerman University of Medical 
Sciences for their full cooperation throughout this endeavor. Additionally, we would like to express our appreciation to the University of Sistan and Baluchestan, where this research was conducted as part of Ms. AJKA's dissertation.

\section{References}

1. Booth FW, Chakravarthy MV, Spangenburg EE. Exercise and gene expression: physiological regulation of the human genome through physical activity. J Physiol. 2002;543(Pt 2):399-411.

2. Glenn AL, Raine A, Yaralian PS, Yang Y. Increased volume of the striatum in psychopathic individuals. Biol Psychiatry. 2010;67(1):52-8.

3. Leahy AA, Mavilidi MF, Smith JJ, Hillman CH, Eather N, Barker D, et al. Review of High-Intensity Interval Training for Cognitive and Mental Health in Youth. Med Sci Sports Exerc. 2020;52(10):222434.

4. Tong L, Shen H, Perreau VM, Balazs R, Cotman CW. Effects of exercise on gene-expression profile in the rat hippocampus. Neurobiol Dis. 2001;8(6):1046-56.

5. Melo CS, Rocha-Vieira E, Freitas DA, Soares BA, Rocha-Gomes A, Riul TR, et al. A single session of high-intensity interval exercise increases antioxidants defenses in the hippocampus of Wistar rats. Physiol Behav. 2019;211:112675.

6. Voss MW, Soto C, Yoo S, Sodoma M, Vivar C, van Praag H. Exercise and Hippocampal Memory Systems. Trends Cogn Sci. 2019;23(4):318-33.

7. Dos Santos JR, Bortolanza M, Ferrari GD, Lanfredi GP, do Nascimento GC, Azzolini A, et al. One-Week High-Intensity Interval Training Increases Hippocampal Plasticity and Mitochondrial Content without Changes in Redox State. Antioxidants (Basel). 2020;9(5).

8. Argandona EG, Bengoetxea H, Ortuzar N, Bulnes S, Rico-Barrio I, Lafuente JV. Vascular endothelial growth factor: adaptive changes in the neuroglialvascular unit. Curr Neurovasc Res. 2012;9(1):72-81.

9. Lou SJ, Liu JY, Chang H, Chen PJ. Hippocampal neurogenesis and gene expression depend on exercise intensity in juvenile rats. Brain Res. 2008;1210:48-55.

10. So JH, Huang C, Ge M, Cai G, Zhang L, Lu Y, et al. Intense Exercise Promotes Adult Hippocampal Neurogenesis But Not Spatial Discrimination. Front Cell Neurosci. 2017;11:13.

11. Hashikawa-Hobara N, Ogawa T, Sakamoto Y, Matsuo Y, Ogawa M, Zamami Y, et al. Calcitonin generelated peptide pre-administration acts as a novel antidepressant in stressed mice. Sci Rep. 2015;5:12559.

12. Singh Y, Gupta G, Shrivastava B, Dahiya R, Tiwari J, Ashwathanarayana M, et al. Calcitonin generelated peptide (CGRP): A novel target for Alzheimer's disease. CNS Neurosci Ther. 2017;23(6):45761.

13. Russo AF. Calcitonin gene-related peptide (CGRP): a new target for migraine. Annu Rev Pharmacol Toxicol. 2015;55:533-52. 
14. Borkum JM. CGRP and Brain Functioning: Cautions for Migraine Treatment. Headache. 2019;59(8):1339-57.

15. Liu XD, Zhang JJ, Wang Y, Yu LC. Inhibitory effects of calcitonin gene-related peptide on long-term potentiation induced in hippocampal slices of rats. Neurosci Lett. 2011;494(1):10-3.

16. Zhai L, Sakurai T, Kamiyoshi A, Ichikawa-Shindo Y, Kawate H, Tanaka M, et al. Endogenous calcitonin gene-related peptide suppresses ischemic brain injuries and progression of cognitive decline. $J$ Hypertens. 2018;36(4):876-91.

17. Dragoni S, Turowski P. Polarised VEGFA Signalling at Vascular Blood-Neural Barriers. Int J Mol Sci. 2018;19(5).

18. Cao L, Jiao X, Zuzga DS, Liu Y, Fong DM, Young D, et al. VEGF links hippocampal activity with neurogenesis, learning and memory. Nat Genet. 2004;36(8):827-35.

19. Bengoetxea H, Argandona EG, Lafuente JV. Effects of visual experience on vascular endothelial growth factor expression during the postnatal development of the rat visual cortex. Cereb Cortex. 2008;18(7):1630-9.

20. Figlin RA, Brown E, Armstrong AJ, Akerley W, Benson AB, 3rd, Burstein HJ, et al. NCCN Task Force Report: mTOR inhibition in solid tumors. J Natl Compr Canc Netw. 2008;6 Suppl 5:S1-20; quiz S1-2.

21. Huang W, Zhu PJ, Zhang S, Zhou H, Stoica L, Galiano M, et al. mTORC2 controls actin polymerization required for consolidation of long-term memory. Nat Neurosci. 2013;16(4):441-8.

22. E L, Lu J, Selfridge JE, Burns JM, Swerdlow RH. Lactate administration reproduces specific brain and liver exercise-related changes. J Neurochem. 2013;127(1):91-100.

23. Zhai L, Liu Y, Zhao W, Chen Q, Guo T, Wei W, et al. Aerobic and resistance training enhances endothelial progenitor cell function via upregulation of caveolin-1 in mice with type 2 diabetes. Stem Cell Res Ther. 2020;11(1):10.

24. Radak Z, Ihasz F, Koltai E, Goto S, Taylor AW, Boldogh I. The redox-associated adaptive response of brain to physical exercise. Free Radic Res. 2014;48(1):84-92.

25. Verboven M, Cuypers A, Deluyker D, Lambrichts I, Eijnde BO, Hansen D, et al. High intensity training improves cardiac function in healthy rats. Sci Rep. 2019;9(1):5612.

26. Hu J, Cai M, Shang Q, Li Z, Feng Y, Liu B, et al. Elevated Lactate by High-Intensity Interval Training Regulates the Hippocampal BDNF Expression and the Mitochondrial Quality Control System. Front Physiol. 2021;12:629914.

27. Batacan RB, Jr., Duncan MJ, Dalbo VJ, Connolly KJ, Fenning AS. Light-intensity and high-intensity interval training improve cardiometabolic health in rats. Appl Physiol Nutr Metab. 2016;41(9):945-52.

28. Wang CT, Mao CJ, Zhang XQ, Zhang CY, Lv DJ, Yang YP, et al. Attenuation of hyperalgesia responses via the modulation of 5-hydroxytryptamine signalings in the rostral ventromedial medulla and spinal cord in a 6-hydroxydopamine-induced rat model of Parkinson's disease. Mol Pain. 2017;13:1744806917691525. 
29. Sun P, Wei S, Wei X, Wang J, Zhang Y, Qiao M, et al. Anger Emotional Stress Influences VEGF/VEGFR2 and Its Induced PI3K/AKT/mTOR Signaling Pathway. Neural Plast. 2016;2016:4129015.

30. Wu H, Lu D, Jiang H, Xiong Y, Qu C, Li B, et al. Simvastatin-mediated upregulation of VEGF and BDNF, activation of the PI3K/Akt pathway, and increase of neurogenesis are associated with therapeutic improvement after traumatic brain injury. J Neurotrauma. 2008;25(2):130-9.

31. Hasbak P, Lundby C, Olsen N, Schifter S, Kanstrup I. Exercise-Induced Calcitonin Gene-Related Peptide (CGRP) Increase during Normoxia and Hypoxia. TheScientificWorldJOURNAL. 2001;1:41-.

32. Ramez M, Rajabi H, Ramezani F, Naderi N, Darbandi-Azar A, Nasirinezhad F. The greater effect of high-intensity interval training versus moderate-intensity continuous training on cardioprotection against ischemia-reperfusion injury through Klotho levels and attenuate of myocardial TRPC6 expression. BMC Cardiovasc Disord. 2019;19(1):118.

33. Cotman CW, Berchtold NC, Christie LA. Exercise builds brain health: key roles of growth factor cascades and inflammation. Trends Neurosci. 2007;30(9):464-72.

34. Guo D, Murdoch CE, Liu T, Qu J, Jiao S, Wang Y, et al. Therapeutic Angiogenesis of Chinese Herbal Medicines in Ischemic Heart Disease: A Review. Front Pharmacol. 2018;9:428.

35. Sparks CA, Guertin DA. Targeting mTOR: prospects for mTOR complex 2 inhibitors in cancer therapy. Oncogene. 2010;29(26):3733-44.

36. Hung CM, Garcia-Haro L, Sparks CA, Guertin DA. mTOR-dependent cell survival mechanisms. Cold Spring Harb Perspect Biol. 2012;4(12).

37. Mitsuoka K, Miwa Y, Kikutani T, Sato I. Localization of CGRP and VEGF mRNAs in the mouse superior cervical ganglion during pre- and postnatal development. Eur J Histochem. 2018;62(4).

38. Tian J, Yang L, Wang P, Yang L, Fan Z. Exogenous CGRP Regulates Apoptosis and Autophagy to Alleviate Traumatic Brain Injury Through Akt/mTOR Signalling Pathway. Neurochem Res. 2020;45(12):2926-38.

39. Downward J. PI 3-kinase, Akt and cell survival. Semin Cell Dev Biol. 2004;15(2):177-82.

40. E L, Burns JM, Swerdlow RH. Effect of high-intensity exercise on aged mouse brain mitochondria, neurogenesis, and inflammation. Neurobiol Aging. 2014;35(11):2574-83.

41. Lloyd BA, Hake HS, Ishiwata T, Farmer CE, Loetz EC, Fleshner M, et al. Exercise increases mTOR signaling in brain regions involved in cognition and emotional behavior. Behav Brain Res. 2017;323:56-67.

\section{Figures}




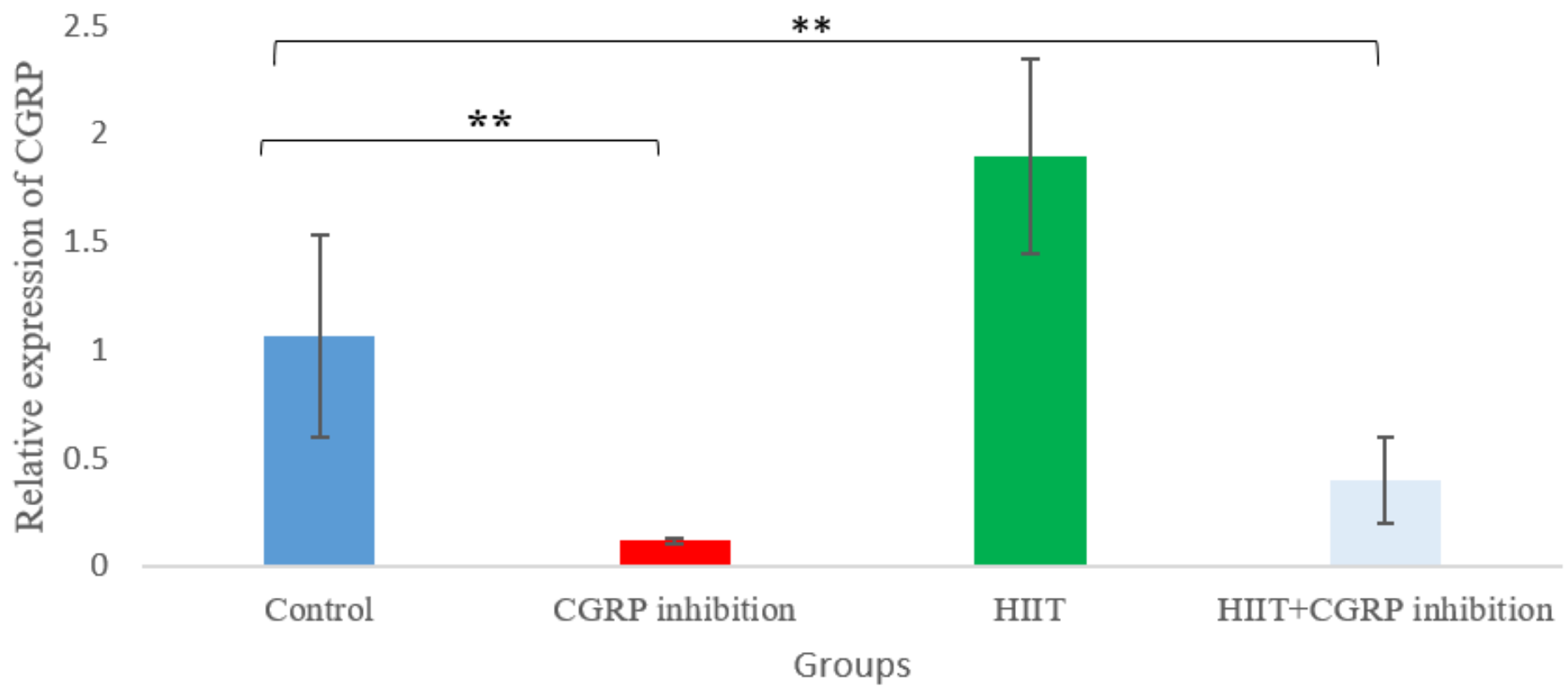

Figure 1

CGRP gene expression comparison in hippocampus tissue across groups ** Significant difference compared to control group $(p<0.05)$.

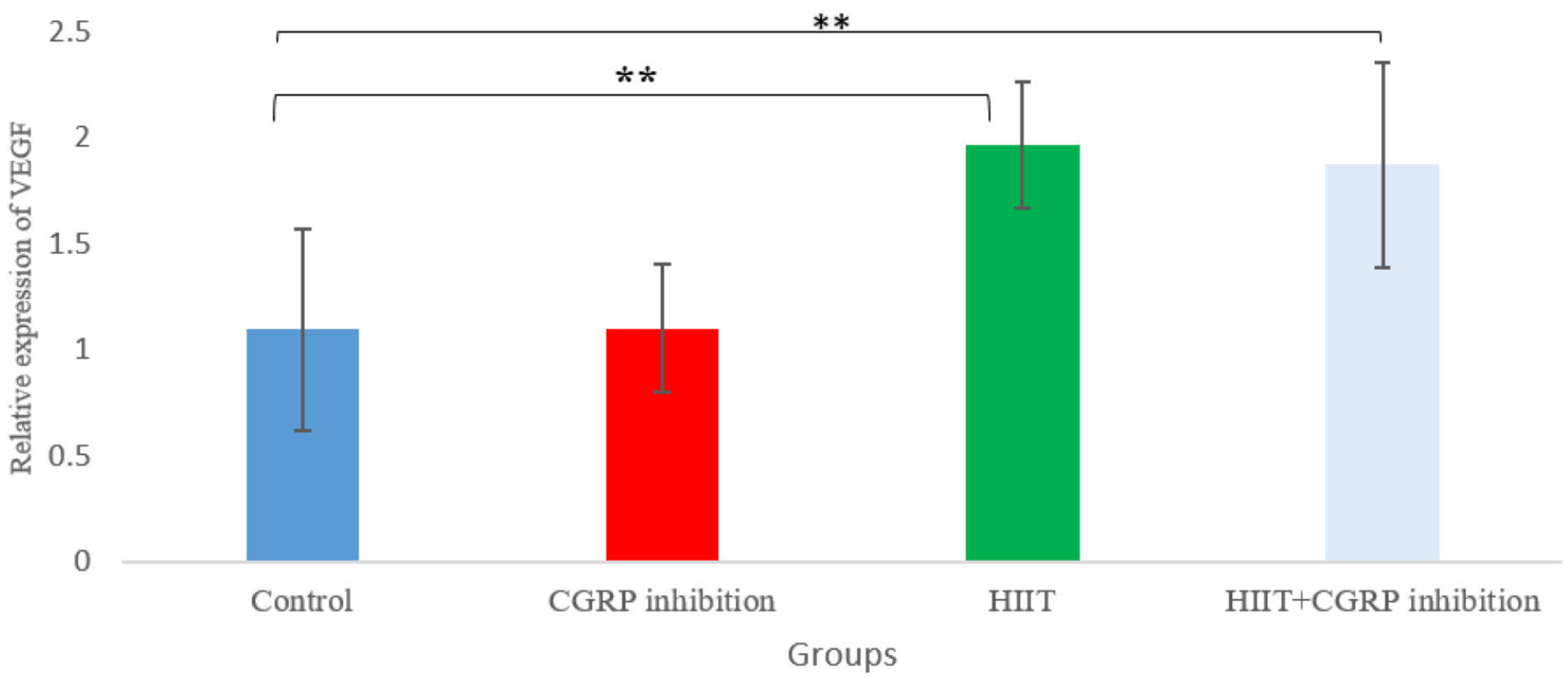

Figure 2

VEGF gene expression comparison in hippocampus tissue across groups 
** Significant difference compared to control group $(P<0.05)$.

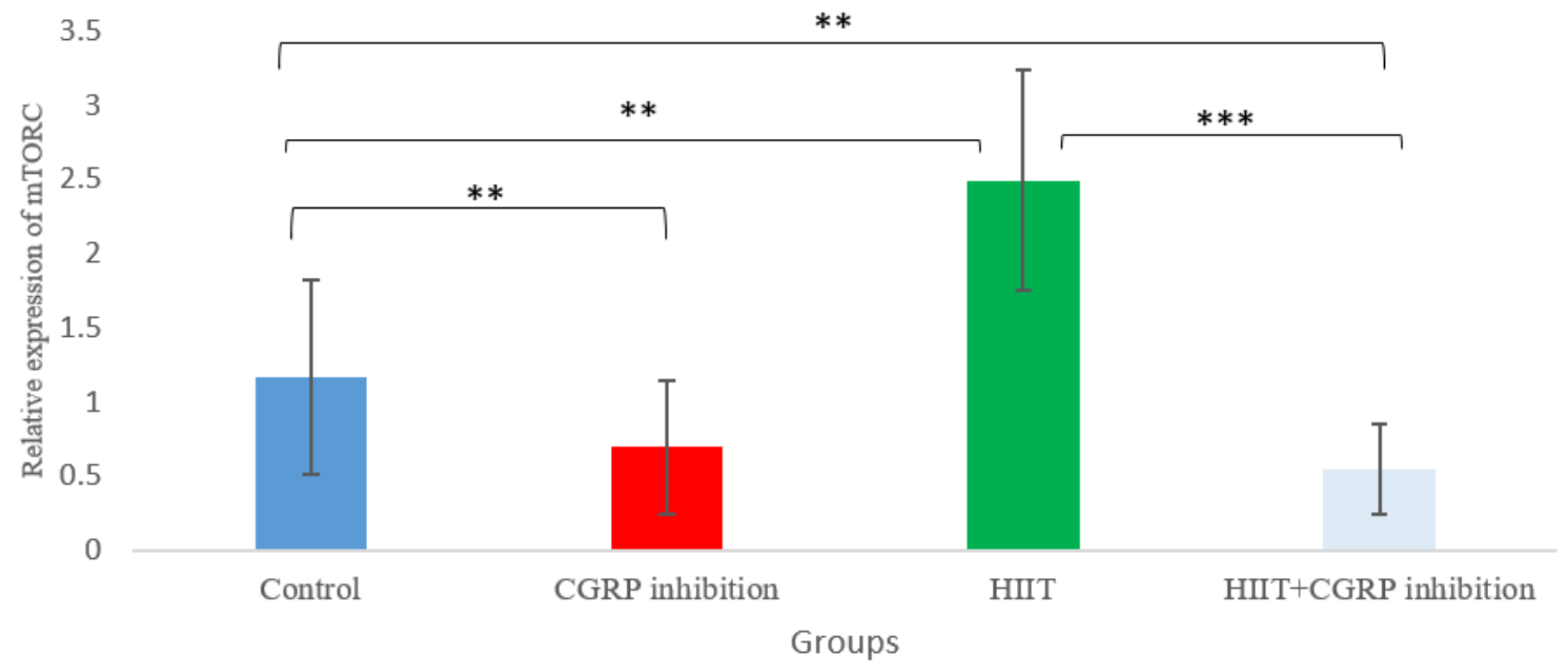

Figure 3

mTORC2 gene expression comparison in hippocampus tissue in study groups

** significant difference compared to control group, ${ }^{* \star *}$ significant difference compared to HIIT group (P $<0.05)$. 